\title{
ANÁLISIS DE LA CALIDAD DE VIDA Y LA EFICIENCIA DE GOBIERNO DESDE LA PERSPECTIVA DE LA COMPETITIVIDAD TERRITORIAL EN LOS MUNICIPIOS DEL ESTADO DE CAMPECHE.
}

\author{
Carlos Pérez Canul*, Román Alberto Quijano García**, Luis Alfredo Arguelles ${ }^{\star * *}$.
}

\begin{abstract}
Pérez-Canul C., Quijano-García R.A., Aguelles L.A. Análisis de la calidad de vida y la eficiencia de gobierno desde la perspectiva de la competitividad territorial en los municipios del Estado de Campeche. Hitos de Ciencias Económico Administrativas 2011;17 (49):115-122.
\end{abstract}

\section{RESUMEN}

Objetivo: Medir la incidencia de la eficiencia de gobierno y la calidad de vida, como factores de la competitividad de los municipios del estado de Campeche del periodo 1998 a 2007 bajo variables estructurales socio-económicas.

Material y método: El estudio presenta un enfoque cuantitativo basado en mediciones numéricas y análisis estadístico que establecen patrones de comportamiento y que derivan en resultados de competitividad, sin embargo éstos no son explicativos de las variables por lo que, aunque no se utilizan como observaciones centrales, existen elementos cualitativos que apoyan el resultado cuantitativo del estudio. La metodología desarrollada proporciona un indicador de la competitividad territorial a nivel municipal considerando elementos socioeconómicos propios del contexto del sureste mexicano.

Resultados: Los resultados muestran que la calidad de vida de los habitantes de los municipios con actividad agropecuaria y menor población es más alta que la obtenida en la capital del estado con la mayor concentración de población y servicios públicos.

Conclusiones: Los valores más altos respecto a la eficiencia del gobierno del estado de Campeche, no necesariamente los obtiene la administración de la capital del estado como normalmente se espera, partiendo del hecho de que la asignación de recursos generalmente se distribuye con base al número de habitantes y a su recaudación.
Pérez-Canul C., Quijano-García R.A., Aguelles L.A. Analysis of the life quality and the government efficiency from the perspective of the territorial competitiveness in the municipalities of the state of Campeche. Hitos de Ciencias Económico Administrativas 2011;17 (49):115-122.

\section{ABSTRACT}

Objective: To measure the impact of the government efficiency and the life quality both as factors of the competitiveness in the municipalities of the state of Campeche from 1998 to 2007 under socioeconomic variable structures.

Material and method: This research shows both a quantitative approach based on numerical measures and a statistical analysis that establish patterns of behavior whose results can be observed in competitiveness. However, these results are not explanatory of the variables, so even though they are not used as main observations, there are qualitative elements that support the quantitative result of this work. The methodology used provides a territorial competitiveness indicator at a municipality level considering socio-economic elements related to the context of the southeast region of Mexico.

Results: The results show that the life quality of the inhabitants of the municipalities that have agricultural activities and less population is higher than the one obtained in those inhabitants who live in the capital of the state where there is more concentration of population and public services.

Conclusions: The highest values regarding the efficiency of the government in the state of Campeche are not necessarily obtained by the administration of the state capital as they are normally expected, due to the fact that the financial allocation is generally distributed according to the number of inhabitants and its financial collection.

Palabras clave: Calidad de vida. Eficiencia de gobierno.

Key words: Life quality. Government efficiency. Regional

Desarrollo regional.. development.

DIRECCIÓN PARA RECIBIR CORRESPONDENCIA:Correo electrónico: rq6715@hotmail.com

* Profesor-Investigador de la Facultad de Contaduría y Administración. Universidad Autónoma de Campeche.

** Profesor-Investigador de la Facultad de Contaduría y Administración. Universidad Autónoma de Campeche.

*** Profesor-Investigador de la Facultad de Contaduría y Administración. Universidad Autónoma de Campeche.

Fecha de recibido: 27 de junio de 2011 Fecha de aceptación: 4 de agosto de 2011. 
I diseño e implementación de políticas públicas por parte del gobierno afecta o beneficia finalmente a los habitantes de la entidad donde se aplican, y se reflejan en la calidad de vida de la población a la cual provee de servicios e infraestructura, según los recursos económicos de los que se dispone. La eficacia con la que se asignan y distribuyen las partidas presupuestarias crea las condiciones para el desarrollo de las actividades económicas de las empresas y los pobladores, y es el resultado de sus acciones lo que determina el nivel de competitividad territorial; su medición ha originado el desarrollo de diversos estudios en nuestro país como «Competitividad Territorial: Ámbitos e Indicadores de Análisis» Sobrino (2005), «Ciudades del siglo XXI: ¿Competitividad o cooperación?» Mendoza (2006) y «Potencial de desarrollo y gestión de la política regional. El caso de Chihuahua» formulado por Gutiérrez (2007), estas investigaciones permiten asumir que existe una relación entre el nivel de competitividad y el desarrollo de una región, aunque un punto importante y complejo es la determinación de indicadores que permitan medir el nivel de competitividad de la región.

\section{Planteamiento del problema.}

Según la información estadística del INEGI (2007) el estado de Campeche, a nivel nacional, presenta un comportamiento económico aceptable con una aportación del $5.6 \%$ al PIB nacional, derivado en gran medida por la presencia de la industria petrolera en el municipio del Carmen, situación que desvirtúa las cifras, ya que nos ubica en una mejor posición contraria a la que refleja la realidad económico-social de los demás municipios del estado. El crecimiento del producto interno bruto (PIB) del estado durante el año 2007 fue de 3.2\% ubicándose en la posición número 17 a nivel nacional de acuerdo al INEGI (2007), por encima de los estados de Tabasco y Yucatán.

En contraste con lo anterior, la medición del Índice de Pobreza Salarial realizado por el Programa de Naciones Unidas para el desarrollo, durante el año 2006, posiciona al estado de Campeche en el lugar número 31 a nivel nacional, esto significa que es una de las entidades en donde el nivel de vida de los habitantes no es el adecuado, tal como se observa en la Tabla I.

Por lo anteriormente mencionado, se requieren de estudios formales que identifiquen y midan cada uno de los elementos que integran la competitividad real del Estado y permita a los distintos interesados el analizar y tomar decisiones para una adecuada planeación económica en busca del desarrollo sostenido que eleve la calidad de vida de sus pobladores.

Preguntas de investigación.

Derivada de la problemática planteada surgen las siguientes interrogantes:

1. ¿Qué estudios se han realizado para medir la competitividad territorial?
2. ¿Cómo incide la eficiencia del gobierno en la calidad de vida, como factor de la competitividad territorial de los municipios del Estado de Campeche?

\section{Objetivos.}

El objetivo general del estudio es medir la incidencia de la eficiencia de gobierno y la calidad de vida, como factores de la competitividad de los municipios del estado de Campeche del periodo 1998 a 2007 bajo variables estructurales socio-económicas.

Los objetivos particulares se describen a continuación:

1. Identificar los elementos significativos que permitan medir la eficiencia de gobierno, así como la calidad de vida como factores en el índice de competitividad territorial de un municipio.

2. Establecer índices que reflejen la posición de la eficiencia de gobierno y la calidad de vida en la determinación de la competitividad municipal del estado de Campeche.

3. Diseñar una metodología para establecer un índice de competitividad municipal y que sea aplicable a cualquier municipio de México.

\section{Limitaciones y delimitaciones del estudio.}

Es necesario considerar para efectos del estudio, que el análisis de la competitividad territorial requiere metodologías distintas respecto al de la competitividad empresarial, en primer lugar porque tiene objetivos distintos y en segundo, por el efecto del desempeño económico de un territorio en el bienestar de su población residente. La presente investigación está limitada por la información que se dispone en las bases de datos de los municipios del estado de Campeche, apoyándose en fuentes oficiales como el Instituto Nacional de Estadística Geografía e Informática (INEGI) y el Consejo Nacional de Población (CONAPO), pues no existe algún organismo o dependencia estatal que desarrolle estudios formales que provean información oficial sobre el tema.

La investigación se realizó en el año 2009 y abarca información estadística del período comprendido del año 1998 a 2007 de los 11 municipios que conforman el Estado de Campeche, México.

\section{MARCO TEÓRICO.}

Existen diversos estudios sobre la competitividad, los más importantes son los llevados a cabo por Porter (1996) y Krugman (1996), que aunque presentan diferentes enfoques, son dos referencias necesarias para entender el ámbito competitivo.

TABLAI POSICIÓN DEL ESTADO DE CAMPECHE SEGÚN SU PIB Y EL ÍNDICE DE POBREZASALARIAL, 2006.

\begin{tabular}{|c|c|c|c|c|}
\hline Entidad & PIB & Posición & IPS & Posición \\
\hline Campeche & $3.2 \%$ & 17 & 0.9074 & 31 \\
\hline
\end{tabular}

Fuente: Programa de las Naciones Unidas para el desarrollo (2006) e INEGI, Encuesta Nacional de Ocupación y Empleo, módulo urbano (2006). 
La economía urbana difiere en muchos sentidos de la economía de un país dentro del campo de la competitividad territorial, por lo que la adaptación del enfoque de ventajas competitivas al contexto local debe abarcar al menos seis elementos: i) los gobiernos locales tienen muy poca injerencia en la formulación de las políticas macroeconómicas de un país; ii) el trabajo y el capital tienen mayor movilidad a escala local; iii) las economías urbanas dependen más del comportamiento de actores y agencias externas a la ciudad; iv) en el contexto local la competitividad de una ciudad se analiza en función del cambio en la participación de alguna variable macroeconómica en el contexto nacional; v) es necesario tomar en cuenta el papel de las políticas regionales del gobierno central, y vi) los gobiernos locales tienden hacia un mayor papel en la promoción del crecimiento económico local (Sobrino, 2005)..

Según la revisión de la literatura efectuada sobre factores empíricos de competitividad local, Sobrino (2005) menciona cuatro aportaciones teóricas, de los cuales se aluden tres de ellas que a juicio de quienes escriben resultan ser las más significativas. A continuación se referirán, en orden cronológico de aparición.

La primera aportación establece que los factores de competitividad local son distintos en el corto y largo plazos; en el primero la base competitiva está determinada por la estructura económica local, carácter y efectividad de sus instituciones y calidad y cantidad de infraestructura, mientras que en el largo plazo la competitividad se sustenta por la adopción de innovaciones tecnológicas y formación de capital humano, por lo que la inversión en capital físico y humano permite consolidar y aumentar las ventajas competitivas locales.

La segunda propuesta establece que la competitividad local es producto de "determinantes económicos y estratégicos». Los determinantes económicos incluyen factores de la producción, infraestructura, localización, estructura económica y amenidades; todas ellas son cuantitativas. Los determinantes estratégicos abarcan efectividad de los gobiernos locales, estrategia urbana, cooperación entre los sectores públicos y privados y flexibilidad institucional; estas son fuerzas cualitativas (Kresl, 1998). Estos elementos se consideraron para conocer la posición competitiva de las 24 áreas metropolitanas más grandes de Estados Unidos en el periodo 1977-1987 y 1987-1992. El rango de competitividad local se obtuvo con un índice ponderado del crecimiento del valor agregado manufacturero, las ventas del comercio al menudeo y los ingresos de los establecimientos de servicios. Cada variable se ponderó de acuerdo con su participación en el valor agregado global de la economía estadounidense.

Por otro lado, las variables explicativas del desempeño económico, o determinantes económicos, fueron ocho: i) crecimiento del ingreso per cápita; ii) centros de investigación/valor agregado manufacturero; iii) crecimiento en el porcentaje de firmas con más de 100 empleados; iv) porcentaje de la fuerza de trabajo con estudios mayores a bachillerato; $v$ ) participación de los trabajadores de investigación y desarrollo en la fuerza laboral total; vi) crecimiento en el número de instituciones culturales, vii) crecimiento en el capital fijo socializado construido por el Estado, y viii) participación de las exportaciones en el producto total. (Kresl y Singh,1999).

Una tercera propuesta se elaboró para estudiar la evolución de las principales ciudades británicas en el periodo comprendido entre 1950 y 1997. El desempeño y competitividad de cada localidad se midió en términos del crecimiento poblacional y las tendencias del empleo y desempleo, en tanto que como factores explicativos del desempeño poblacional y ocupacional propusieron un modelo de cambio y participación. Los resultados muestran tendencias sistemáticas y persistentes en el sistema urbano británico, en el cual las grandes conurbaciones han sido las principales perdedoras, mientras que las de menor tamaño, en el sur del país, las cercanas a Londres y las New Towns han sido las principales ganadoras. Asimismo, el estudio menciona que la penetración de importaciones, el decline de actividades tradicionales y la emergencia de nuevos tipos de actividad, sobre todo del sector terciario moderno, explican los resultados del análisis de cambio y participación (Begg, 2002).

Ahora se menciona la contribución de Sobrino (2002 y 2003) al conocimiento sobre la naturaleza competitiva de las ciudades en México. «La competitividad no es un atributo que puede ser medido directamente, sino se debe adoptar algún indicador de la misma; en este caso se eligió el crecimiento del valor bruto de la producción (VBP) industrial, comercial y de servicios. La justificación de estos sectores como indicadores de competitividad económica local es la siguiente: el crecimiento en las ventas manufactureras refleja inversiones que se traducen en ampliación de su base exportadora y generación de empleo; el crecimiento de los ingresos derivados del comercio y servicios reflejan el dinamismo demográfico de la ciudad, incremento en el ingreso de los habitantes y atractividad de no residentes para ir de compras, acceder a servicios de educación y salud o por motivos turísticos recreativos y culturales».

Para poner en operación la propuesta anterior de ventajas competitivas locales y explorar el desempeño competitivo de las ciudades del estudio, se construyó una base de datos con los siguientes indicadores cuantitativos, ventajas competitivas empresariales: i) Productividad parcial del trabajo; ii) Remuneración promedio al personal ocupado (sueldos); iii) Intensidad del capital, o relación capital-trabajo (capital); iv) Tamaño promedio de los establecimientos (tamaño), y v) Porcentaje del personal ocupado en la industria maquiladora de exportación respecto a la demanda ocupacional de la estructura productiva local. Las tres primeras estiman la eficiencia microeconómica de los establecimientos manufactureros (Ballance 1987; González y Mariña 1992; Roper 1998) y la cuarta es una acotación de la concentración técnica (Utton, 1975). 
En 1980 las 39 ciudades del estudio concentraron $80.1 \%$ del VPB industrial, comercial y de servicios nacional, aportación que se redujo a $79.4 \%$ en 1998 , lo que indica que la crisis económica se manifestó con mayor claridad en las estructuras económicas de las ciudades más importantes del país. En 1998 la participación de las ciudades aumentó $84.3 \%$, indicando un nuevo proceso de concentración territorial ante la mejoría económica registrada en el contexto nacional. El indicador de competitividad se estableció, en primer lugar como el cambio en la participación absoluta (CPA) de una ciudad en el VBP nacional del sector en cuestión.

En Síntesis y de acuerdo a Sobrino (2005), la posición competitiva de las ciudades durante la década de los ochenta muestras que el mejor desempeño económico local se sustentó en el sector servicios, en la región frontera norte y en ciudades con los mayores tamaños de población después de la ciudad de México. En contraposición, los mayores efectos de la crisis económica se presentaron en la ciudad de México y sus localidades vecinas más cercanas -Toluca y Pachuca, o en otras palabras, en la emergente conformación megalopolitana del centro del país.

\section{METODOLOGÍA.}

Enfoque.- El estudio presenta un enfoque cuantitativo basado en mediciones numéricas y análisis estadístico que establecen patrones de comportamiento y que derivan en resultados de competitividad, sin embargo éstos no son explicativos de las variables por lo que, aunque no se utilizan como observaciones centrales, existen elementos cualitativos que apoyan el resultado cuantitativo del estudio.

Tipo.- El estudio es descriptivo, ya que únicamente pretende medir información de manera conjunta sobre las variables de referencia.

Diseño.- Estudio no experimental, pues las variables presentadas en él no son alteradas de ninguna forma durante la realización del mismo. El diseño del estudio es longitudinal de evolución de grupo (cohort).

Contexto.- En cuanto a la temporalidad del estudio se recolectaron datos de los años 1998 al 2007, se tomaron como base los años 2000 y 2005, puesto que en estos años se elaboraron los Censos Económicos. En otros años la información resultó ser en extremo limitada y repetitiva (con base a los censos antes mencionados). La investigación se ubica territorialmente en la ciudad de San Francisco de Campeche, México.

\section{Participantes en el estudio.}

Universo y muestra.- El estudio realizado fue de tipo longitudinal de evolución de grupos (cohorts), definido como un examen de los cambios a través del tiempo en subpoblaciones o grupos específicos, los cuales están vinculados siempre de alguna manera o son identificados por una característica común, la cual, en nuestro caso, es la época. Los participantes del mismo fueron los 11 municipios del estado de Campeche: Calakmul, Calkiní, Campeche, Candelaria, Carmen, Champotón, Escárcega, Hecelchakán, Hopelchén, Palizada, y Tenabo.

Instrumento.- Para desarrollar el Instrumento se siguió el método estadístico mediante una secuencia de procedimientos para el manejo de los datos cuantitativos de la investigación. El manejo de datos tiene por propósito la comprobación de una o varias consecuencias verificables de las preguntas de investigación. Se diseño una matriz de factores que contempla los municipios del Estado de Campeche, el período y las variables a investigar, ver Tabla II.

Procedimiento para la recolección de datos.

Con base a la información del International Institute for Management Development (IMD) (2004), del World Economics Forum (WEF) (2008) y otros organismos, se
TABLA II. FACTOR «N» PONDERACIÓN DE CADA SOLUCIÓN SOBRE CADA INCONVENIENTE.

\begin{tabular}{|c|c|c|c|}
\hline Año & Variable 1 & Variable “n” \\
\hline 2000 & $x$ & $x$ \\
\hline 2000 & $x$ & $x$ \\
\hline 2000 & $x$ & $x$ \\
\hline
\end{tabular}

Fuente: Elaboración propia.

determinó los factores a utilizar en el estudio y que se presentan en la Tabla III.

Cabe señalar que, si bien estas sugerencias no son tomadas íntegramente para nuestro estudio (en afán de contextualización regional), sirven de base para la elaboración del Índice, pues nos permiten establecer el peso ponderado que tendrán los factores a la hora de calcular el mismo.

La asignación del peso de cada factor en el ICM es un punto importante de la investigación y se decidió con base a la información originada en la tabla I, asignando un peso mayor a los factores competitivos más utilizados y un peso menor a factores propios de la región. Para el estudio se definieron diferentes variables englobadas en 6 factores, los cuales fueron la base de los resultados que dieron origen al Índice de Competitividad Municipal (ICM) descritos en la Tabla IV.

Descripción de los procesos de recolección de datos:

Se consultaron principalmente los anuarios estadísticos del INEGI correspondientes a cada municipio de la entidad; en el CONAPO se revisaron datos económicos y proyecciones de población, que ayudaron a completar las tablas. Dado que en algunos casos la información era inexistente en estos organismos, se consultaron libros emitidos por el gobierno del Estado, como «Situación Demográfica del Estado de Campeche», emitido por el Consejo Estatal de Población. 
PÉREZ-CANUL C., QUIJANO-GARCÍA R.A., ARGUELLES L.A.

TABLA III. ELEMENTOS DE COMPETITIVIDAD CONSIDERADOS POR DIFERENTES ORGANISMOS E INSTITUCIONES A NIVEL MUNDIAL.

\begin{tabular}{|c|c|c|c|c|c|c|c|c|c|}
\hline Factor & WEF & IMD & Chile & Colombia & Ecuador & $\begin{array}{l}\text { Reino } \\
\text { Unido }\end{array}$ & Filipinas & Europeo & Campeche \\
\hline Institucionalidad & $x$ & $x$ & $x$ & $x$ & $x$ & & $x$ & & $x$ \\
\hline Infraestructura & $x$ & $x$ & $x$ & $x$ & $x$ & & $x$ & $x$ & $x$ \\
\hline Macroeconomía & $x$ & $x$ & $x$ & $x$ & $x$ & $x$ & $x$ & $x$ & $x$ \\
\hline Salud & $x$ & $x$ & $x$ & $x$ & & & & & $x$ \\
\hline Educación & $x$ & $x$ & $x$ & $x$ & $x$ & $x$ & $x$ & $x$ & $x$ \\
\hline Mercado laboral & $x$ & $x$ & & $x$ & & $x$ & & & $x$ \\
\hline Mercado financiero & $x$ & $x$ & $x$ & $x$ & $x$ & & $x$ & & \\
\hline Preparación tecnológica & $x$ & $x$ & $x$ & $x$ & $x$ & & & $x$ & \\
\hline Mercado & $x$ & $x$ & & & $x$ & $x$ & & & \\
\hline Sofisticación de negocios & $x$ & & & $x$ & $x$ & & $x$ & & \\
\hline Innovación & $x$ & $x$ & $x$ & $x$ & $x$ & $x$ & & $x$ & \\
\hline Recursos naturales & & $x$ & $x$ & $x$ & & & $x$ & & \\
\hline
\end{tabular}

Fuente: Elaboración propia.

\section{TABLA IV. FACTORES CONSIDERADOS PARA EL ÍNDICE DE COMPETITIVIDAD MUNICIPAL.}

\begin{tabular}{|c|c|}
\hline Factor: Recursos humanos & Factor: Calidad de vida \\
\hline $\begin{array}{l}\text { - \% de población analfabeta. } \\
\text { - \% de población sin primaria completa de } 15 \text { años o } \\
\text { más. } \\
\text { • \% población ocupada con ingreso de hasta } 2 \text { salarios } \\
\text { mínimos. } \\
\text { - Conflictos de trabajo por cada } 100,000 \text { habitantes. } \\
\text { - Alumnos por maestro en primaria. } \\
\text { - Instituciones de educación por casa } 100,000 \\
\text { habitantes. }\end{array}$ & $\begin{array}{l}\text { - \% de población con servicio de agua potable. } \\
\text { - \% población con servicio eléctrico. } \\
\text { •\% de población con servicio de alcantarillado. } \\
\text { - Clínicas por cada 100,000 habitantes. } \\
\text { - Doctores por cada 100,000 habitantes. } \\
\text { - Habitantes por km². } \\
\text { - Tasa de mortalidad. } \\
\text { - Población derechohabiente entre población } \\
\text { total. }\end{array}$ \\
\hline Factor: Condiciones de la economía & Factor: Infraestructura \\
\hline $\begin{array}{l}\text { - Población total. } \\
\text { - Tasa de inflación. } \\
\text { - Índice de marginación. }\end{array}$ & $\begin{array}{l}\text { - Km de camino pavimentado por cada } \\
100,000 \text { habitantes. } \\
\text { - Viviendas entre población total. } \\
\text { - Bibliotecas por cada } 100,000 \text { habitantes. }\end{array}$ \\
\hline Factor: Ambiente de negocios & Factor: Eficiencia de gobierno \\
\hline $\begin{array}{l}\text { - Homicidios por cada } 100,000 \text { habitantes. } \\
\text { - Crecimiento en los homicidios. } \\
\text { - Delitos del fuero común. } \\
\text { - Asegurados por el IMSS entre población total. }\end{array}$ & $\begin{array}{l}\text { - Egresos totales entre población total. } \\
\text { - Gasto en obra pública por cada } 100,000 \\
\text { habitantes. } \\
\text { - Ingresos por impuestos entre población } \\
\text { total. } \\
\text { - Ingresos por impuestos entre total de } \\
\text { ingresos. } \\
\text { - Ingresos totales entre población total. } \\
\text { - Crecimiento en ingresos totales entre } \\
\text { población total. } \\
\text { - Subsidios, transferencias y ayudas por cada } \\
\text { - Ingo,000 habitantes. }\end{array}$ \\
\hline
\end{tabular}

Fuente: Elaboración propia, con datos proporcionados por el Dr. Antonio Serrano. 
Diseño del índice.- El diseño del modelo se llevó a cabo definiendo los factores y variables que se debían incluir, así como las calificaciones y los pesos respectivos. Las Variables incluidas en cada factor recibieron una puntuación de acuerdo a su posición.

Números Índice.- Los números índices son indicadores de varios aspectos de la industria y el comercio, y nos permiten comparar rápidamente eventos relacionados con periodos de tiempo y espacio. Tales números variarán con la fecha y también con el área del país a la que se refieran. Los números índices normalmente comienzan con una base de 100 en un tiempo en particular para el país entero. Se verá que los números índices, proporcionan series de tiempo, y están sujetas a análisis en cuanto a movimientos de tendencia y estacionales.

En este caso, se tienen elementos que son de distinta clase. Estos datos por si solos no pueden ser sumados, y por tanto promediados. Para asignar una calificación, primeramente se obtuvo una media de cada variable por factor.

Así mismo, se calculó la desviación estándar de los mismos datos, lo que nos permitió establecer rangos tanto a la derecha como a la izquierda de la media, los datos de cada municipio se fueron ubicando en esos rangos y se les otorgó una calificación, dependiendo de la naturaleza de la variable (ya sea que a mayor número de unidades mayor calificación o viceversa). El siguiente paso fue obtener una calificación general, para lo cual se elaboró la suma de las calificaciones de cada variable y la determinación de los datos adicionales necesarios (la media y la desviación estándar). Con base a esto se repitió el mismo procedimiento antes mencionado, por lo que se obtuvo una media de medias que nos permitió calificar al factor.

Un punto importante en la elaboración del Índice fue la designación de los pesos que se basaron en información relativa a los factores del International Institute for Management Development, de Lausana, Suiza, en su «Anuario de Competitividad Mundial» (2004), así como de los factores utilizador por el Foro Económico Mundial (2008). Tabla V.

Una vez asignado el peso a cada factor se procedió a establecer la fórmula que nos permitió calcular el Índice de Competitividad Municipal, compuesto por el resultado ponderado de cada factor con base a su peso:

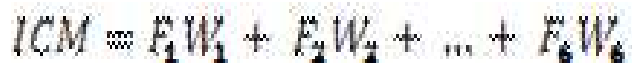

Donde: $F$ es el resultado de cada Factor y $W$ es el peso específico del factor.
El resultado de cada factor se determina mediante el promedio de calificaciones obtenidas en las variables incluidas es dicho factor.

\section{RESULTADOS.}

De acuerdo a la metodología establecida para la creación del Índice de Competitividad Municipal (ICM) y la evaluación de la incidencia de los factores eficiencia de gobierno y calidad de vida en las unidades de análisis (municipios) se obtuvieron los siguientes resultados para el año 2000, ver Tabla VI.

El análisis de la información de la tabla $V$ permite comparar la calificación ponderada según el procedimiento diseñado

\section{TABLA V. FACTORES PONDERADOS PARA EL ÍNDICE DE COMPETITIVIDAD MUNICIPAL.}

\begin{tabular}{|c|c|}
\hline Factor & Peso \\
\hline Calidad de vida & $40 \%$ \\
\hline Eficiencia del gobierno & $30 \%$ \\
\hline Infraestructura & $10 \%$ \\
\hline $\begin{array}{l}\text { Condiciones de la } \\
\text { economía }\end{array}$ & $10 \%$ \\
\hline Recursos humanos & $5 \%$ \\
\hline Ambiente de negocio & $5 \%$ \\
\hline Total & $100 \%$ \\
\hline
\end{tabular}

Fuente: Elaboración propia.

\section{TABLA VI. FACTORES EFICIENCIA DE GOBIERNO, CALIDAD DE VIDAE} ÍNDICE DE COMPETITIVIDAD TERRITORIAL AÑO 2000.

\begin{tabular}{|l|c|c|c|}
\hline Municipio & $\begin{array}{c}\text { Eficiencia de } \\
\text { gobierno }\end{array}$ & $\begin{array}{c}\text { Calidad } \\
\text { de vida }\end{array}$ & ICM \\
\hline Palizada & 10.00 & 10.00 & 8.45 \\
\hline Campeche & 8.75 & 7.40 & 7.94 \\
\hline Carmen & 7.50 & 8.60 & 7.55 \\
\hline Champotón & 7.50 & 7.40 & 7.06 \\
\hline Calkiní & 5.00 & 1.00 & 3.25 \\
\hline Escárcega & 5.00 & 6.20 & 5.01 \\
\hline Holpechén & 5.00 & 5.00 & 4.86 \\
\hline Calakmul & 3.00 & 1.00 & 2.00 \\
\hline Candelaria & 3.00 & 3.00 & 3.65 \\
\hline Tenabo & 3.00 & 5.00 & 4.76 \\
\hline Hecelchakán & 1.00 & 3.00 & 3.40 \\
\hline
\end{tabular}

Fuente: Elaboración propia. 
para la obtención del ICM (que incluye todos los elementos de la tabla IV) y adaptado para el entorno particular del estado de Campeche perteneciente a la región sureste de México. Según los resultados obtenidos para el año 2000 el municipio con la puntuación más alta en competitividad, es el de Palizada el cual no concentra la mayor población del estado de Campeche y cuya actividad económica primordial pertenece al sector agropecuario, mientras que la capital del mismo (Campeche) se posiciona en segundo sitio y el municipio del Carmen se ubica en el tercero. En el otro extremo los municipios de Calkiní y de Calakmul, tienen los valores más pequeños de competitividad, aunque en el último caso probablemente se deba a su reciente nombramiento como municipio, pues anteriormente era parte del municipio de Hopelchén. La puntuación más alta de los dos factores (eficiencia de gobierno y calidad de vida) los obtiene Palizada que coincide con la puntuación más alta también del ICM, sin embargo Campeche (que ocupaba el segundo lugar del ICM) en este caso, ocupa el segundo sitio en eficiencia de gobierno pero se ubica en el tercer puesto en calidad de vida de la población que habita en la capital del estado. En el caso de Carmen que ocupa el tercer sitio en el ICM permanece en ese puesto respecto a la eficiencia de gobierno y ocupa en segundo sitio en el factor calidad de vida. La puntuación más baja en eficiencia de gobierno y calidad de vida se asignó a Calakmul precedido por Calkiní, que igual ocupan los últimos sitios en el ICM.

Al aplicar la misma metodología para el año 2005 se obtuvieron resultados plasmados en la Tabla VII.

\section{CONCLUSIONES.}

Discusión de resultados.

Los hallazgos del estudio en cada uno de los años evaluados, arrojan que los valores más altos respecto a la eficiencia del gobierno del estado de Campeche, no necesariamente los obtiene la administración de la capital del estado como normalmente se espera, partiendo del hecho de que la asignación de recursos generalmente se distribuye con base al número de habitantes y a su recaudación, lo que pone en duda la eficiencia y maximización por parte de las autoridades gubernamentales de las partidas presupuestales asignadas. Por el contrario los municipios dedicados a la actividad agropecuaria y que no concentran la mayor población fueron los que obtuvieron el valor más alto como Palizada (2000) y Candelaria y Escárcega (2005), situación que no se esperaba.

Respecto al factor calidad de vida no se esperaba que un municipio preponderantemente con actividad ganadera como Palizada, fuera el que tuviera el puntaje más alto en esta área en el año 2000 aunque fuera desplazado en 2005 por Carmen, Campeche y Escárcega; en este caso los dos primeros son los municipios más grandes y con mayor concentración de habitantes lo que les garantiza una mayor participación en los recursos pero el tercer sitio es ocupado por Escárcega otra población preponderantemente agropecuaria.

El factor calidad de vida confirmó que en el caso del Carmen la presencia de la paraestatal PEMEX ha influido en forma positiva en el desarrollo de este
Al comparar los resultados de la Tabla VI, se obtuvo que las condiciones de competitividad de los municipios del Estado variaron después de 5 años, ocupando el primer sitio Campeche (actividad comercial), Carmen (actividad petrolera) y Escárcega (sector agropecuario), respecto al factor Calidad de vida; pero la eficiencia de su gobierno los ubica en el puesto 11,3 y 2 respectivamente; siendo ocupado el primer lugar por el municipio de Candelaria (sector agropecuario). Los últimos puestos los obtuvieron Calakmul y Hecelchakán en términos de calidad de vida de los habitantes y Calakmul, Calkiní, Campeche y Hecelchakán en el factor eficiencia de gobierno.

La Tabla VI señala que al considerar todos los elementos contemplados en la Tabla IV para determinar el ICM, los resultados de los primeros lugares de calidad de vida se invierten y es el municipio de Escárcega el más competitivo, ubicándose Carmen en el segundo y la capital del estado en el tercer sitio. Los últimos sitios del ICM para 2005 son ocupados por Calakmul (10) y Hecelchakán en el puesto 11.
TABLA VII. FACTOR: EFICIENCIA DE GOBIERNO Y CALIDAD DE VIDA E ÍNDICE DE COMPETITIVIDAD TERRITORIAL AÑO 2005.

\begin{tabular}{|l|c|c|c|}
\hline Municipio & $\begin{array}{c}\text { Eficiencia de } \\
\text { gobierno }\end{array}$ & $\begin{array}{c}\text { Calidad } \\
\text { de vida }\end{array}$ & ICM \\
\hline Candelaria & 10.00 & 3.00 & 5.89 \\
\hline Escárcega & 8.75 & 10.00 & 8.15 \\
\hline Carmen & 7.50 & 10.00 & 7.84 \\
\hline Palizada & 7.50 & 6.60 & 6.76 \\
\hline Champotón & 6.25 & 8.20 & 7.16 \\
\hline Hopelchén & 5.00 & 1.00 & 2.94 \\
\hline Tenabo & 5.00 & 6.60 & 6.07 \\
\hline Calakmul & 3.70 & 1.00 & 2.71 \\
\hline Calkiní & 3.70 & 3.00 & 3.76 \\
\hline Campeche & 3.70 & 10.00 & 7.57 \\
\hline Hecelchakán & 1.00 & 1.00 & 2.43 \\
\hline
\end{tabular}

Fuente: Elaboración propia. 
municipio aunque los costos sociales sean considerados altos. Al contrastar la puntuación de la calidad de vida contra la eficiencia de gobierno de este municipio se encontró que los resultados alcanzados por la administración municipal del período de estudio muestran que la calidad de vida mejoró para los habitantes del municipio pero no por las acciones de su gobierno pues éste factor mantiene el mismo valor en 2000 y 2005 lo que lo posiciona en el cuarto sitio, y que ratifica su dependencia económica de la actividad petrolera.

Igualmente se halló, contrario a lo esperado, que de acuerdo al Índice de competitividad municipal y que agrupa todos los factores de la metodología propuesta para el Estado de Campeche, son los municipios con actividades primordialmente agropecuarias Palizada (2000) y Escárcega (2005), quienes reportan el nivel más alto de competitividad. En contraste el municipio de Calakmul en los dos periodos evaluados, permanece en los últimos sitios siendo una localidad con vastos recursos naturales, pero que no están siendo explotados de forma sustentable sin agredir el medio ambiente y en beneficio de su población.

\section{Recomendaciones.}

Los resultados obtenidos confirman la necesidad de proponer e implementar una metodología de medición de la competitividad como exigencia de la globalización que sufre la economía a nivel mundial y de la cual no están exentos los municipios del país. Es deseable complementar este estudio desarrollando entrevistas o cuestionarios con ciudadanos, delegados de dependencias federales, estatales y municipales e incluso con secretarios de gobierno que emitieran su opinión sobre la calidad de vida alcanzada y la política económica del estado, su aplicación y los resultados alcanzados por esta.

Se requiere un organismo estatal que tenga como función monitorear de forma permanente y autónoma los resultados económicos de las decisiones del gobierno del estado en cuanto a los parámetros para la distribución y transparencia en la asignación de recursos, y que esta información sea considerada en el diseño de la política económica por lo menos del estado de Campeche.

\section{REFERENCIAS}

Ariighi, G. (1994). The Long Twentieth Century . Money, Power and the origins of our times, Verso, Londres.

Begg, I. (2002). «Introduction» en lain Begg (ed.). Urban Competitiveness, The Policy Press, Bristol, pp.1-10.

Boisier, S. (1994). Postmodernismo territorial y Globalización: regiones pivótales y regiones virtuales. Ciudad y Territorio. Estudios Territoriales. , 102. Pp. 597-608.

Budd, L. (1998). Territorial Competition and Globalization: Scylla and Changbdis of Europen Cities. Urba Studies , 35 (4), pp. 663-685.

Gutierrez, L. E. (2007). Potencial de desarrollo y gestión de la política regional. El caso Chihuahua. Frontera Norte,7-35.

Kresl, P. (1998). La respuesta de la economía urbana al Tratado de Libre Comercio de América del Norte :Planificar para la competitividad. Economia, Sociedad y Territorio, 1 (4), pp. 695-722..

Krugman, P. (1991). International Economics. Trade and Policy,Harper Collins Publisher, Nueva York.

Malecki, E. (1997). Technology and Economics Development. Londres: Logman.

Mendoza, E. C. (2006). Ciudades del Siglo XXI: ¿Competitividad o Cooperación? Gestión y Política Pública , 201-206.

Porter, M. (1991). Ventaja Competitiva de las Naciones. España. Ed. Vergara.

Porter, M. (1993). Estrategia Competitiva. México: CECSA.

Porter, M. (1996). Competitive Advantage, Agglomeration Economies and Regional Policy. International Regional Science Review 19, 1-2.

Scholte, J. (2000). Globalization. A critical Introduction. Palgrave, Nueva York.

Sobrino, J. (2005). Competitividad Territorial. Ámbitos e indicadores de análisis. Economia, Sociedad y Territorio , 123-183.

Utton, M. (1975). La concentración Industrial. Madrid: Alianza Editorial. 